Audit of section 2 and section 3 mental health act paperwork in Derby inpatient psychiatric units using an audit tool by Mason et al. (2012)

\author{
Abigail Pearson ${ }^{1 *}$, Andrew Horton ${ }^{1}$ and Mike Akroyd ${ }^{2}$ \\ ${ }^{1}$ Former Clinical Leadership Fellow at Derbyshire Healthcare NHS \\ Foundation Trust, CT1 Doctor at Sheffield Health and Social Care \\ and ${ }^{2}$ Consultant Psychiatrist, Derbyshire Healthcare NHS \\ Foundation Trust \\ ${ }^{*}$ Corresponding author.
}

doi: 10.1192/bjo.2021.294

Aims. Complete an audit of Section 2 and Section 3 Mental Health Act Paperwork in Derby Inpatient psychiatric units using an audit tool developed in a study by Mason et al. (2012). Background. The 1983 Mental Health Act enables doctors approved on behalf of the Secretary of State under Section 12 to be able to make recommendations for the detention of individuals with a mental health problem where the degree and/or nature, and associated risk to that person's health or safety, or that of others, makes inpatient care necessary. For the detention and the associated deprivation of their liberty to be lawful, it is necessary that the clinical situation meets certain criteria as outlined in the Mental Health Act.

Method. Ward status was reviewed for each inpatient ward in Derby and the first five patients alphabetically, who were detained under sections 2 or 3 were selected. The Mental Health Act medical recommendation documents were reviewed according to the necessary criteria, using an assessment tool generated from a study by Mason et al. in 2012 'Compulsion under the Mental Health Act 1983: audit of the quality of medical recommendations'. A junior colleague was trained to analyse Mental Health Act paperwork using the audit tool. Medical recommendations were reviewed and rated as 'clear', 'implied' or 'none' for each criterion.

Result. Evidence of a mental health problem and the nature or degree of illness was well documented. Evidence regarding why informal admission was not appropriate was also reasonable but with room for improvement. Poor compliance was evident mostly in relation to the justification related to risk to health, safety or others, the lowest clearly documented percentage of these appear to be regarding health.

Conclusion. From analysing the documentation, often written justification incorporated general safety as a whole; however health and safety are identified by the mental health act as separate criterion requiring clear justification of each. In a number of occasions people failed to identify which of the three risk categories were relevant for the patient. Potential criticisms of this audit include the subjective nature of the interpretation of clearly explained and implied and that data analysis was completed by a non-section 12 approved doctor. Data were presented at the local weekly academic teaching to raise awareness of the results and a recommendation was made for the subject to be included in the junior doctor induction.

Are blood tests being performed for new inpatient admissions to a psychiatric hospital as recommended by RCPsych guidelines?

Isabella Piper*

Leverndale Psychiatric Hospital

${ }^{*}$ Corresponding author.

doi: 10.1192/bjo.2021.295

Aims. Severe mental illness (SMI) has a significant impact on a person's physical health and mortality. There is a 10-25-year life expectancy reduction in patients with SMI. The majority of deaths are due to physical health conditions. The Royal College of Psychiatry (RCPsych) sets out a standard that new inpatient admissions to Mental Health Services should have routine blood tests performed within 24 hours of admission, unless they have had a recent blood test. The aim of this audit was to review whether blood tests were performed either in the 48 hours preceding admission or the 48 hours after admission to Leverndale Hospital.

Method. Clinical records were reviewed for new inpatient admissions to two general adult wards over a four-month period.

Result. 79 patients were admitted $(\mathrm{M}=39, \mathrm{~F}=40$, Age: $18-62$ years old). $70 / 79$ (89\%) had blood tests performed within the 48-hour timeframe. 5/79 (6\%) had a blood test performed after 48 hours of their admission. 4/79 (5\%) did not have a blood test. The blood tests performed varied. 51/75 (68\%) patients had at least one abnormal blood test. The yield of abnormal blood results ranged from $2 \%$ for thyroid function tests to $35 \%$ for a full blood count. Conclusion. This audit has established that the majority of patients had blood tests performed within the 48-hour timeframe. This could be improved by setting up an electronic reminder to prompt the clinician to perform a blood test at 24 hours as per RCPsych guidance if one had not yet been done. The blood tests performed varied. RCPsych guidance does not specify which blood tests should be done. A further scope for this audit could be to review the clinical significance of abnormal blood results to develop a standard set of blood tests for admission.

\section{Venous thromboembolism (VTE) risk assessment completion in psychiatric inpatients}

Harriet Powell ${ }^{1 \star}$ and Josie Jenkinson ${ }^{2}$

${ }^{1}$ Ashford and St Peter's NHS Trust and ${ }^{2}$ Surrey and Borders

Partnership NHS Foundation trust

${ }^{\star}$ Corresponding author.

doi: $10.1192 /$ bjo.2021.296

Aims. To audit VTE risk assessment compliance across psychiatric inpatient wards at three different sites within Surrey and Borders Partnership NHS Foundation Trust (SABP), and to highlight the importance of completing VTE risk assessments for psychiatric inpatient safety and care as set out by NICE guidelines (2019).

Method. Numbers of VTE risk assessments completed (within 24 hours, and those completed any time during inpatient stay) and VTE risk assessments not completed were collected via SABP electronic mental health records. Percentage compliance for each ward and hospital involved in the study were calculated. Chi square statistical $t$ tests were conducted using Excel to check for associations between type of ward (older adult and working age) and VTE risk assessment completion.

A total of 3004 patients were included in the study. Ages ranged from 18-82 years of age, and both males and females included in the study. A total of 2060 were working age (WA) patients (aged 18-64 years) and 944 were older adults (OA) (aged $>65$ years).

Result. Across all three sites, more than $90 \%$ of all inpatients admitted between May 2018 and October 2020 did not have a formal VTE risk assessment completed. Across all sites, less than $1 \%$ of all inpatients had a completed VTE risk assessment done within 24 hours, as recommended by the NICE guidelines. Older Adult wards showed better compliance with VTE risk assessment completion with $38 \%$ of patients on one OA ward having had a completed VTE risk assessment, and 28\% on another completed OA ward. Being admitted to an OA ward 
was strongly associated with VTE risk assessment completion $(\mathrm{p}<0.05)$.

Conclusion. OA wards have hosted QI programmes with regards to VTE risk assessment which may be why VTE risk assessment was more likely to have been completed on OA wards. VTE risk assessment compliance overall is inadequate across all sites included in the study. Recommendations include further education for all ward staff on how, why and when VTE risk assessment should be completed, greater accessibility of an improved VTE risk assessment form and for QI initiatives on OA wards to be rolled out on WA wards. These findings have been presented and discussed at regional Trust teaching days, and this audit will be repeated in one year.

\section{An audit of liaison service provision in Aneurin Bevan University Health Board}

Jennifer Rankin* and Heledd Espley

Aneurin Bevan University Health Board

${ }^{*}$ Corresponding author.

doi: 10.1192/bjo.2021.297

Aims. Aneurin Bevan University Health Board (ABUHB) isundertaking a review of the Mental Health Liaison Service provision within it's acute general hospitals. The current liaison service is a small nurse led team which is available between $8 \mathrm{am}$ and midnight. ABUHB has recently opened a new Specialist Critical Care Hospital with the liaison service moving into a new base. A new model of care has been developed across the healthboard which has stretched the Liaison Service across several sites. Therefore, the liaison service may need to expanded to be able to provide high quality and timely care across a wide geographical area. The audit aims to idenitfy areas in which the liaison service is performing well in while identiying areas that need improvement. This audit may provide a focus for recommendations to enhance the current liaison provision.

Method. The liaison service was audited against RCPsych Psychiatric Liaison Accreditation Network (PLAN) quality standards. PLAN identified eighteen functions of a liaison team and provided details of quality standards within each function. These standards are either considered essential, expected or desirable. An accredited service is expected to meet $100 \%$ of essential standards, $80 \%$ of expected standards and $60 \%$ of desirable standards. Data were taken from a combination of sources including ABUHB policies, service managers and senior clinicians within both mental health and acute services.

Result. When comparing the current liaison service provision in ABUHB, $30 \%$ of essential standards were not met and $21 \%$ were only somewhat met. Particular domains that were identifed as needing improvement included policies and procedures and urgent and emergency mental health care. $36 \%$ of expected standards were met with $41 \%$ not met. Notable domains that the service was performing poorly in included governance; induction, and providing teaching and support to acute colleagues. $89 \%$ of desirable standards were not met.

Conclusion. The audit idenitifed that the current liaison service fails to meet core standards set out by RCPsych. This audit provides quantitative data to demonstrate that the liaison service is in need of improvement and investment. As a result, enhaving the current liaison service is now a priortity for the health board. A business case is being developed to consider enhancing the liaison service with a view to developing a Consultant led multidisciplinary team. The business case can use PLAN quality standards to make recommendations for improvements to the service.

\section{A clinical audit to investigate polypharmacy and interactions in inpatients in an old age psychiatric ward}

\author{
Mohan Rathnaiah $^{1 *}$, Beth Brailsford ${ }^{2}$ and Nisha Mokashi ${ }^{3}$ \\ ${ }^{1}$ Derbyshire Healthcare NHS FT; ${ }^{2}$ UHDB NHS Founation Trust and \\ ${ }^{3}$ Nottinghamshire Healthcare NHS Foundation Trust \\ ${ }^{\star}$ Corresponding author.
}

doi: $10.1192 /$ bjo.2021.298

Aims. To identify any problematic polypharmacy in the patient records of those staying in Cherry Ward, an old age psychiatric unit at Highbury Hospital, Nottingham in the calendar year 2018. Background. Multi-morbidity is defined as more than one longterm medical condition in a single individual and is a factor that is closely associated with polypharmacy, the use of multiple medications concurrently. Appropriate and Problematic are the two classifications of polypharmacy outlined by the King's Fund report, the first describing optimised evidenced-based pharmacological management of comorbidities and the latter to label prescribed medications whose use is not in the best interests of the patient. The risk of drug interactions and adverse drug reactions is increased with polypharmacy, and frail elderly patients are particularly at risk of the side-effects of psychotropic medications used in the management of mental health disorders. Guidelines highlight this group as a key party to be identified when searching for at-risk people.

Method. The electronic records of those admitted and discharged from Cherry Ward in 2018 were reviewed in the period spanning January to May 2019, and the first forty-three patients were analysed in Microsoft Excel using criteria based on the King's fund report and the Medscape and BNF (British National Formulary) drug interaction tools. The Medscape drug interaction checker was used for initial screening; the complete medication list for each patient was entered into it and the number of interactions was displayed with advice on severity. If necessary, the individual interactions for each specific medication could also be crossreferenced in the BNF using the extensive lists provided for each drug. These are also graded from mild to severe.

Result. On discharge, $69.7 \%$ (thirty patients) met the criteria for being at higher risk of polypharmacy. One patient became at higher risk of polypharmacy during admission, and another two stepped down from meeting the criteria on admission but not on discharge. Thirty-one of the forty-three patients had at least one interaction recorded; $18.6 \%$ (eight patients) had at least one potentially severe interaction.

Conclusion. A substantial proportion of patients in Cherry ward in 2018 were at a higher risk of polypharmacy, reflecting current practice as outlined in the King's Fund report. Problematic polypharmacy is common among older patients hospitalised with psychiatric illness. Recommendations include use of an automated electronic system to investigate and flag up problematic polypharmacy and severe medication interactions.

\section{Cervical screening in assertive outreach team patients}

Hannah Reynolds*, Samaila Bello and Hanna Leech

Birmingham and Solihull Mental Health NHS Foundation Trust ${ }^{\star}$ Corresponding author.

doi: 10.1192/bjo.2021.299

Aims. To assess the uptake of cervical screening in patients under Birmingham and Solihull Assertive Outreach Teams; this 